\title{
Automated Planning with Multivariate Shape Descriptors for Fibular Transfer in Mandibular Reconstruction
}

\section{AUTHOR(S):}

Nakao, Megumi; Aso, Shimpei; Imai, Yuichiro; Ueda, Nobuhiro; Hatanaka, Toshihide; Shiba, Mao; Kirita, Tadaaki; Matsuda, Tetsuya

\section{CITATION:}

Nakao, Megumi ... [et al]. Automated Planning with Multivariate Shape Descriptors for Fibular Transfer in Mandibular Reconstruction. IEEE Transactions on Biomedical Engineering 2017, 64(8): 1772-1785

\section{ISSUE DATE:}

2017-08

URL:

http://hdl.handle.net/2433/217346

\section{RIGHT:}

(c) 2016 IEEE. Personal use of this material is permitted. Permission from IEEE must be obtained for all other uses, in any current or future media, including reprinting/republishing this material for advertising or promotional purposes,

creating new collective works, for resale or redistribution to servers or lists, or reuse of any copyrighted component of this work in other works.; This is not the published version. Please cite only the published version.; この論文は出版社版 でありません。引用の際には出版社版をご確認ご利用ください。 


\title{
Automated Planning with Multivariate Shape Descriptors for Fibular Transfer in Mandibular Reconstruction
}

\author{
Megumi Nakao, Member, IEEE, Shimpei Aso, Yuichiro Imai, Nobuhiro Ueda, Toshihide Hatanaka, Mao Shiba, \\ Tadaaki Kirita, and Tetsuya Matsuda, Member, IEEE
}

\begin{abstract}
Objective: This paper introduces methods to automate preoperative planning of fibular segmentation and placement for mandibular reconstruction with fibular flaps. Methods: Preoperative virtual planning for this type of surgery has been performed by manual adjustment of many parameters, or based upon a single feature of the reconstruction. We propose a novel planning procedure formulated as a non-convex minimization problem of an objective function using the multilateral shape descriptors. Results: A retrospective study was designed and 120 reconstruction plans were reproduced using computed tomography images with oral surgeons. The proposed automated planning model was quantitatively compared with both the existing model and the surgeons' plans. Conclusion: The results show that the developed framework attains stable automated planning that agrees with the surgeons' decisions. Significance: This method addresses trade-off problems between symmetric reconstruction and restoration of the native contour of the mandible.
\end{abstract}

Index Terms-Automated planning, Shape descriptor, Surgical process modeling, Fibular transfer surgery, Mandibular reconstruction

\section{INTRODUCTION}

$\mathbf{M}$ ANDIBULAR reconstruction is generally performed to prevent postoperative aesthetic and masticatory disturbances after mandibular resection in patients with mandibular gingival cancer. This reconstruction is performed by grafting a vascularized autologous fibular flap, anastomosing blood vessels in the flap to blood vessels in the neck [1], [2]. The main advantage of this technique is that transplantation of autologous bone with blood vessels promotes bone engraftment better than artificial bone [3]. Creating an in-depth preoperative reconstruction plan is important for accurate and stable reconstruction of the patient's original facial appearance and for mandible function. Preoperative planning requires determination of optimal placement of the fibular flap (or segment) in the mandibular resection area and identification of the fibular harvesting site and number of segments required. However, it is difficult to gain a clear understanding of the three-dimensional (3D) shape of the mandible postreconstruction and to determine the appropriate segmentation

M. Nakao, S. Aso and T. Matsuda are with Graduate School of Informatics, Kyoto University, Kyoto, Yoshida-Honmachi, Sakyo, Kyoto, JAPAN. e-mail: megumi@i.kyoto-u.ac.jp

Y. Imai is with Department of Oral and Maxillofacial Surgery, Rakuwakai Otowa Hospital, Yamashina, Kyoto, 607-8062, JAPAN.

N. Ueda, T. Hatanaka, M. Shiba and T. Kirita are with Department of Oral and Maxillofacial Surgery, Nara Medical University, Kashihara, Nara, 634-0813, JAPAN.

Manuscript received August 19, 2016; revised October 4, 2016. and optimal placement of the fibular graft based on 2D Xrays and computed tomography (CT) images. If the fibular segment does not match the shape of the mandibular resection area, additional intraoperative bone cutting is necessary, which prolongs surgery [4], [5]. Mandibular reconstruction via fibular transfer is more difficult than reconstruction with artificial bone. Most decisions regarding harvesting of bone grafts, segmentation, and placement in mandibular reconstruction depend on the surgeon's knowledge and experience.

Many studies have focused on the efficacy of preoperative planning systems that use 3D-CT images in mandibular reconstruction [6]-[11]. In addition to the method in which the mandible is reconstructed with two or three fibular segments, various placements of the fibular segments and more complex reconstruction, referred to as "double barrel" fibular flaps, have also been studied [12]. Virtual planning systems enable interactive simulation of segmentation and placement of fibular segments within a 3D space [4], [10], [13], [14]. However, it is often difficult for surgeons and dental technicians to obtain the intended position because minute adjustments of the location and orientation of segments must be performed manually by inputting numerical parameters or with a mouse. Objective preoperative planning that does not depend on individual subjectivity or manual operation is needed to ensure procedure standardization. Quantifying the requirements and developing a framework to formulate reconstruction plans for individual patients based on objective quantitative indices will bring us a step closer to resolving these problems.

Several studies have attempted to quantify planning and explore indices for evaluation of the shape of the hip joint, femur, and tibia [15]-[17]. Studies examining shape have also been conducted for the purpose of diagnosing craniosynostosis [18], [19]. However, quantitative indices for mandibular reconstruction have not been sufficiently studied. Reported attempts to automate surgical planning include a system for planning hip replacement [20]. However, segmentation and placement of fibular segments for mandibular reconstruction is highly flexible, and the shape of the mandible varies from person to person [21], [22]. Therefore, quantification of the requirements for mandibular reconstruction and development and automation of systems that can handle complex reconstructions are necessary.

The aim of this study was to automate the process of segmentation and placement of fibular segments during preoperative planning for mandibular reconstruction. We propose to 
formulate the planning procedure as a non-convex minimization problem of an objective function using multilateral shape descriptors. Because surgeons emphasize the restoration of facial appearance, quantification of differences between the local shape of the patient's original mandible and the reconstructed mandible has been considered. Furthermore, mirror-symmetric distances have been considered for postoperative mandibular symmetry. Using multiple shape descriptors to design objective functions, multilateral evaluation of the mandible before and after reconstruction can be achieved. Applying a series of optimization algorithms to 3D-CT images of individual patients should automate most preoperative planning and produce more objective reconstruction plans.

We designed a retrospective study to confirm the efficacy of the automated planning methods. Several oral surgeons and dental technicians participated in an experiment to simulate preoperative planning based on CT data of past cases; a total of 120 different reconstruction plans were produced. We compared the proposed framework by quantitatively comparing our automated planning model with both the existing model and the surgeons' plans. The issues with stable automated planning that considers both restoration of the patient's native mandible and symmetric reconstruction were quantitatively investigated in the experiments.

\section{RELATED WORK}

In the field of computer-assisted surgery, hip replacement arthroplasty [16], [17] is one surgical procedure in which automation of statistical shape models and preoperative planning has actively progressed. Preoperative planning for hip replacement arthroplasty requires determination of the correct size, placement, angle, and other aspects of the artificial joint to prevent postoperative dislocation, wear, damage, loosening, and other associated problems. Previous studies have reported systems that incorporate the surgeon's medical knowledge and the patient's morphological features obtained from medical images to automatically calculate the optimal placement of artificial joints [20], [23]. However, while predetermination of the size and placement of an artificial joint (which is a fixed size and type) may be problematic in hip replacement arthroplasty, preoperative planning for mandibular reconstruction using autologous bone requires identification of the fibular harvesting site and the angle of the resection lines. Moreover, reconstruction can sometimes involve three or more fibular segments, and the shape variability of the mandible [22], offering a higher degree of freedom during surgical planning.

Virtual surgical planning based on computer-aided design is increasingly popular for mandibular reconstruction. Steinbacher and Tarsitano et al. reviewed the details of head and mandibular reconstruction models using preoperative planning systems [24], [25]. The use of 3D printers to manufacture models from plans has led to the development of metal fittings, referred to as surgical guides [26], [27]. Numerous attempts have been made to accurately harvest fibular grafts using these guides [2], [4], [10]. 3D-CT images have also been used to design the shape of titanium plates for fixing grafted bone to the mandible. Khalifa et al. [28] used computer-aided design to project a mirror image of the contralateral mandible onto the mandibular defect and then designed titanium trays based on this shape. Several recent clinical studies have investigated the accuracy of virtual planning by comparing the pre-surgical plan with reconstruction results [29]-[31]. [32] analyzed the biomedical behavior of the mandible after marginal resection from a mechanical perspective using finite element analysis, while [33] investigated the shape of the soft-tissue flap transplanted with the bone flap. These recent studies address major issues in clinical outcomes and patient satisfaction. [34] developed a haptics-assisted planning system that incorporates bone resection, fibula design, recipient vessel selection, pedicle and perforator location selection, and skin paddle configuration. [35] demonstrated the cost advantages of preoperative planning.

To optimize the patient-specific procedure, the virtual plan should be quantitatively assessed as completely and accurately as possible during the preoperative planning stage. Previous reports have described methods to quantitatively evaluate skull shape in craniosynostosis [18] and the use of statistical shape models [19] in its diagnosis. [36] developed a planning model for face-jaw-teeth transplantation. However, to the best of our knowledge, few studies have focused on statistical shape modeling for mandibular reconstruction via fibular transfer or on the automation of preoperative planning. A recent report by Brown et al. [37] presents new classification criteria for mandibular defects after oncological resection. Seiler et al. [22] provided a data-driven, multi-scale description of mandibles and analyzed anatomical shape variability. Raith et al. [38] introduced statistical shape representation with anatomy-related topology to reconstruct the missing parts. In clinical studies, mirror-image-based 3D templates for mandibular and maxillary reconstruction have been used to ensure symmetrical reconstruction [28], [39], [40]. However, these models do not evaluate the difference between preoperative and postoperative facial appearance. From an aesthetic point of view, restoration of the patient's native mandible is an essential issue in fibular transfer surgery, but quantitative analysis of the personalized reconstruction procedures has not been well studied. Studies on shape analysis include a report by Nakao et al. [13], [14], in which the authors propose an interactive planning system with indices for the quantitative evaluation of the shape of reconstructed mandibles when different numbers of fibular segments are used. Although interactive planning is useful for quantifying the surgeon's knowledge and experience [41], objective planning that does not depend on individual subjectivity is needed for procedure standardization.

This paper introduces a completely systematic approach unlike previous interactive planning systems, and proposes a novel automated framework that formulates reconstruction plans for individual patients by exploring multidimensional shape descriptors. Although many issues that clinically influence the reconstruction, such as the length of the vascular pedicle [34], size of the segments, location of the segments, and oncological resection margins [24], [33], should be considered, we focused on the outer shape of the bony contour as a first step in automated planning. 


\section{METHODS}

\section{A. Automated fibular transfer planning}

This section describes methods to automate the process of segmentation and placement of fibular segments during preoperative planning for mandibular reconstruction using fibular grafts, a process that previously required manual adjustment of many parameters and decisions based on the surgeon's knowledge and experience. To automate this process, shape descriptors that multilaterally quantify the post-reconstruction shape of the mandible were introduced based on the native shape of the patient's mandible. Furthermore, objective functions based on these shape descriptors were designed, and placements that minimized these functions were identified to achieve automation of planning.

1) Mandibular reconstruction model: The automated surgical planning framework in this study was designed based on geometric definitions from surgical planning models [14], [41] used in interactive mandibular reconstruction planning systems. The mandibular resection area is planned first, with consideration of the most appropriate margins for removal of cancerous lesions or reconstruction of mandibular defects. The resection area is determined using the boundary surfaces in the volumetric mandibular image $I$, as shown in Fig. 1a. Mandibular reconstruction with fibular segments can be efficiently simulated using connection points between the fibular segments and corresponding virtual planes. In this study, we introduced a set of $N+1$ reconstruction parameters $\boldsymbol{\theta}=\left[\boldsymbol{\theta}_{0}, \boldsymbol{\theta}_{1}, \ldots, \boldsymbol{\theta}_{N}\right]$ to formulate fibular transfer plans with $N$ fibular segments, where the reconstruction parameter $\boldsymbol{\theta}_{i}$ represents the position and the orientation of the boundary surface. For the $N$ fibular segments, controllable planes are used to define the resection area and osteotomy lines for fibular shaping. Here, $\boldsymbol{p}_{0}$ and $\boldsymbol{p}_{N}$ on the virtual planes represent the 3D positions of the connection points between the patient's native mandible and the fibular segments. The other points, $\boldsymbol{p}_{N}(i=1, \ldots, N-1)$, are used as connection points between two fibular segments. Three-dimensional rotation of the plane is described with a $4 \mathrm{D}$ vector $\boldsymbol{q}_{i}$, i.e., quaternion. The setting of the connection point $\boldsymbol{p}_{i}$ and the quaternion $\boldsymbol{q}_{i}$ gives a simple form for the boundary surface (Fig. 1b). Based on this formulation, the reconstruction parameter $\boldsymbol{\theta}_{i}$ is described as follows:

$$
\boldsymbol{\theta}_{i}=\left[\boldsymbol{p}_{i}, \boldsymbol{q}_{i}\right] .
$$

The corresponding virtual planes $\hat{S}_{h}(h=0, \ldots, 2 N-1)$ are placed on the fibular image $I_{f}$ to define the fibular segments. Fig. 1c shows three fibular segments defined by six planes: $\hat{S}_{0}, \ldots, \hat{S}_{5}$ (termed a three-segment procedure in this paper). A two-segment procedure is similarly defined using four virtual planes. Using the reconstruction parameter $\boldsymbol{\theta}_{i}$, each plane $\hat{S}_{h}$ is mapped to the corresponding plane $S_{h}$ in the resection area of the mandible. Fig. 1a shows the simulation results with the oriented fibular image $I_{f}^{\prime}$ that is segmented and transformed with the reconstruction parameter set $\boldsymbol{\theta}$ from the fibular CT image $I_{f}$. Thus, a reconstruction plan is uniquely defined by $\boldsymbol{\theta}$. This scheme also enables the user to refine the plan while

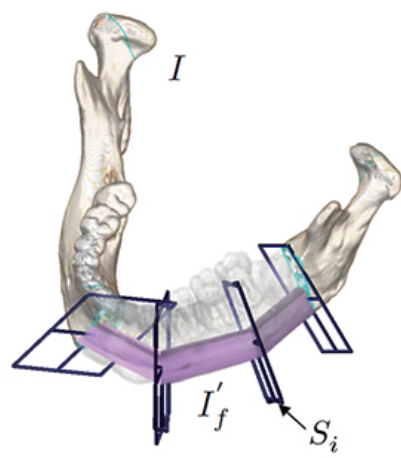

(a)

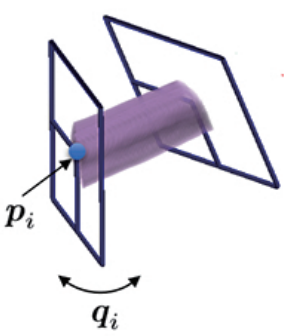

(b)

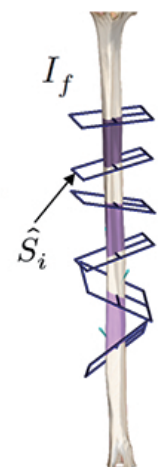

(c)
Fig. 1. Geometrical description of fibular transfer simulation. (a) typical planning example with three fibular segments superimposed on the resection area, (b) reconstruction parameters for the virtual plane, and (c) native fibular volume annotated with the corresponding virtual planes.

observing simulation results on both mandibular reconstruction and fibular osteotomy. Direct volume manipulation and visualization of multiple objects [42], [43] allow interactive editing of the surgical plan, including virtual resection, without the need for a surface modeling process.

2) Objective function for automated planning: Using the aforementioned mandibular reconstruction planning models, the number of fibular segments $N$ used in reconstruction and the local optimization reconstruction parameter $\boldsymbol{\theta}$ were calculated, based on individual patient 3D-CT images of the mandible $I$ and fibula $I_{f}$. The reconstruction parameters $\boldsymbol{\theta}_{0}$ and $\boldsymbol{\theta}_{N}$, which determine the resection area, were determined by the surgeon, taking into account tumor location and size. In addition, the initial value for the remaining reconstruction parameter $\boldsymbol{\theta}_{i}(1 \leq i \leq N-1)$ is uniquely defined by the linear interpolation of $\boldsymbol{\theta}_{0}$ and $\boldsymbol{\theta}_{N}$. For the above subjects, an objective function $E$ that quantifies reconstruction planning was designed, and the fibular transfer planning process was formulated as the minimization problem described by

$$
\boldsymbol{\theta}^{*}=\underset{\boldsymbol{\theta}}{\operatorname{argmin}} E\left(I, I_{f}, N, \boldsymbol{\theta}\right) .
$$

Here, the objective function $E$ must reflect the decisions made based on the surgeon's medical knowledge and experience. However, as explained in the previous section, the mandibular reconstruction procedure has not yet been sufficiently standardized. Fig. 2 shows four possible examples of simple reconstruction plans designed by surgeons. The upper images show two-segment procedures with two different placements of the connection point $\boldsymbol{p}_{1}$. This variation results from differences in the geometrical features of the resection area, the patient's native mandible, and the fibular segments. In (a), it is conceivable that the fibular segments have been placed to minimize variations from the patient's native mandibular shape, whereas in (b), the placement of fibular segments has resulted in a larger depression in the mental region of the mandible. This placement could have been chosen to achieve mandibular symmetry post-reconstruction. The red and blue annotations show the difference in degree of symmetry. 


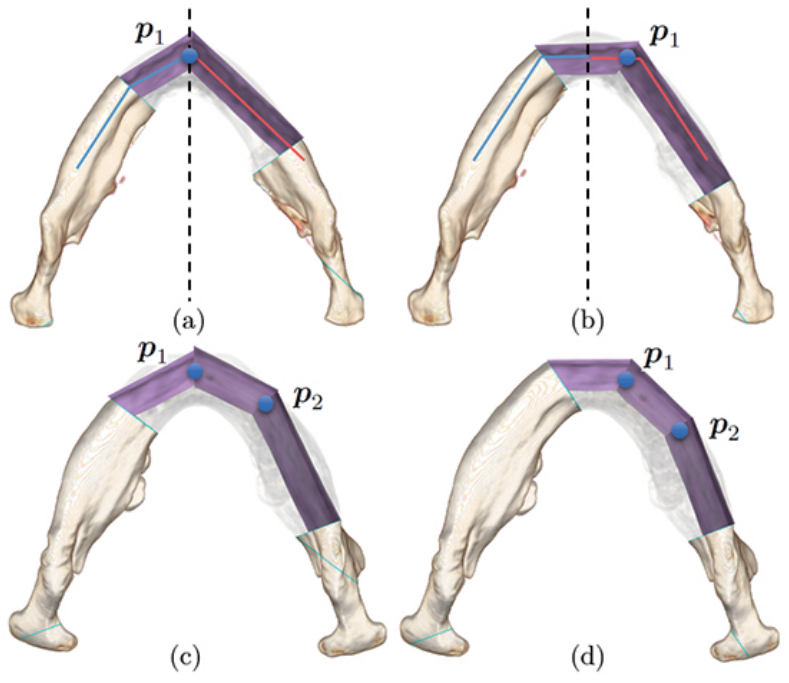

Fig. 2. Fibular transfer simulation examples. (a,b) manually defined planning results with different placements of the connection point, (c, d) other planning results with three fibular segments.

The case shown in the lower panels has an anatomically similar resection area, but in this case surgeons selected a three-segment procedure. The connection points $\boldsymbol{p}_{1}$ and $\boldsymbol{p}_{2}$ in (c) are different from those in (d). From a geometrical viewpoint, the three-segment procedure generally reconstructs the curved shape of the native mandible better than the twosegment procedure. Despite this advantage, the two-segment procedure remains a good choice for actual surgery because of segment size and the required surgery time. These decisions have been empirically made based on the estimated visual appearance, and it is quantitatively unclear which placement leads to better reconstruction.

Based on the decision-making trends in conventional manual planning, we introduced two shape descriptors to evaluate the shape of the mandible after reconstruction of a wide resection area, regardless of individual differences in mandibular shape, by referencing shape evaluation indices for chin reconstruction proposed in previous studies [14] and a symmetric transformation approach previously used for reconstruction of mandibular defects [28], [39], [40]. Because surgeons emphasize the restoration of facial appearance, shape distance $E_{s}$ was introduced as an index to quantify the difference in local shape between the patient's original mandible and the reconstructed mandible. Furthermore, to address mandibular symmetry, the mirror-symmetric distance $E_{r}$ was introduced as an index to quantify the difference in the surface shape between the left and right mandible after reconstruction. By designing an evaluation function based on these two shape descriptors, it was possible to multilaterally appraise the mandible before and after reconstruction.

In automated planning with three fibular segments, the dimensions of the parameters subject to optimization, $\boldsymbol{p}_{i}$ and $\boldsymbol{q}_{i}$, have 9 and 12 degrees of freedom, respectively, giving parameter $\boldsymbol{\theta}$ a total of 21 dimensions. This suggests that calculation time and stability could be problematic during optimization. If we assume that this system will be used in

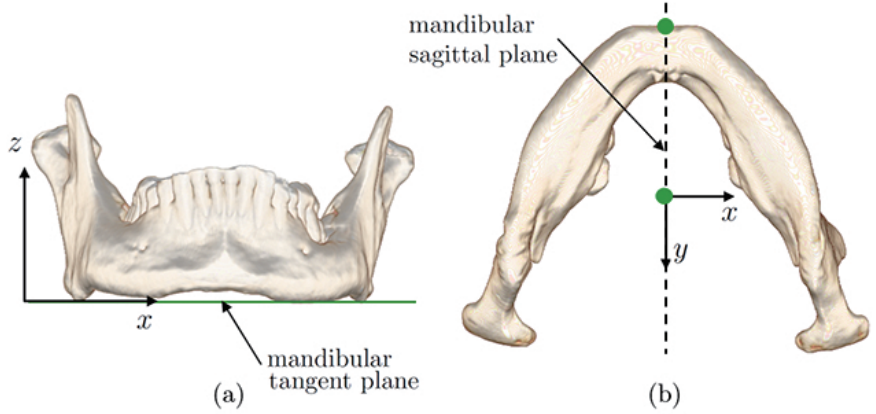

Fig. 3. Mandibular coordinates uniquely defined on the mandible image. (a) Mandibular tangent plane and (b) mandibular sagittal plane.

preoperative planning, the solution will need to be calculated within a few minutes. The control surface rotation parameter $\boldsymbol{q}_{i}$ was therefore uniquely determined with the linear interpolation of the rotation parameters of the adjacent control surface based on the positions of the corresponding connection points. An optimization algorithm was also introduced to shorten the calculation time required to derive the minimum solution for the objective function.

\section{B. Shape descriptors}

1) Mandibular coordinates: In this section, the coordinate system used for automation will be explained. The posture of the patient during measurement affects the relative position and orientation of the mandible in the 3D-CT images. For quantitative evaluation of the reconstruction plans, a unique 3D coordinate system should be determined for the individual mandibular image $I$. In general, surgeons carefully consider reconstruction based on the shape of the chin and the lower edge of the native mandible. Based on these observations, a mandibular tangent plane uniquely defined for the 3D surface of the mandible is created. Fig. 3a shows an example of a mandibular tangent plane placed at the lower edge of the mandible. This reference plane can be computed as a ground plane when a rigid object drops to the ground and reaches a stable state. In this paper, the normal vector of the mandibular tangent plane is referred to as the $z$ axis. The $y$ axis of the mandibular coordinate system is defined by drawing a straight line connecting an origin point, determined by projecting the position of the mandibular center of gravity onto the mandibular tangent plane, and the mandibular midline, as shown in Fig. 3b. The defined $y z$ plane is referred to as the mandibular sagittal plane. The $x$ axis is uniquely determined as the cross product of the $y$ and $z$ axes. The orthogonal coordinate system obtained with the above procedure is defined as the mandibular coordinates for patient-specific 3D imaging.

2) Shape distance: This section describes the details of the shape distance $E_{s}$ and its calculation method. This index is intended to quantify the shape error between the outer surface of the patient's native mandible and that of the post-transfer fibular segments. Because the surfaces of the mandible and fibula are curved and have a complex local shape, formulation by approximation using secondary curved surfaces and other simple parameterization is not appropriate. Hence, the outer 


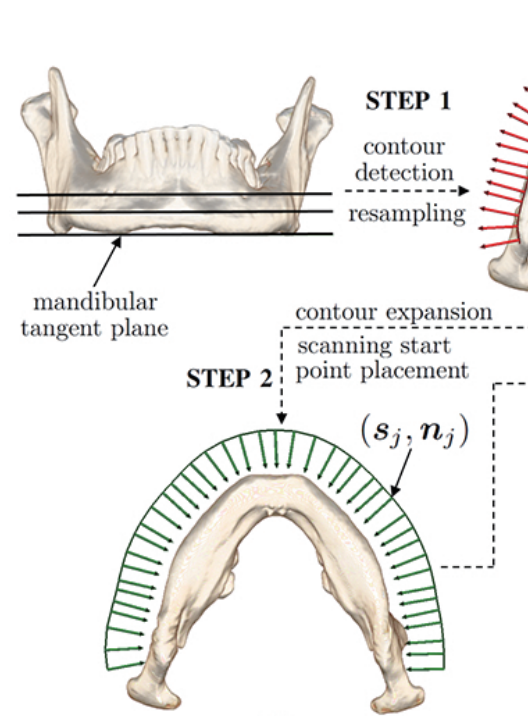

(b)

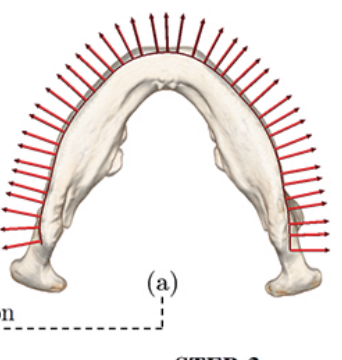

STEP 3

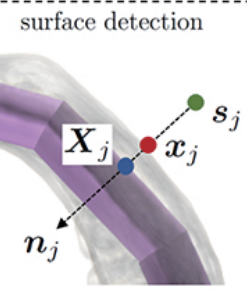

(c)
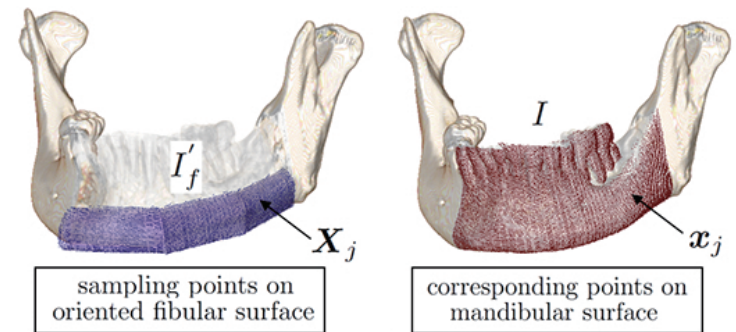

corresponding points on mandibular surface

(d)

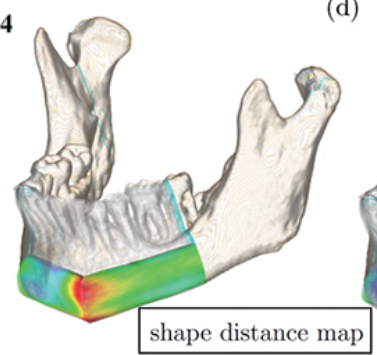

(e)

Fig. 4. Shape distance computation and mapping on the oriented fibular segments. (a) Mandibular contour detection. (b) Contour expansion and scanning start point placement. (c) Detection of the point pair of $\boldsymbol{x}_{j}$ and $\boldsymbol{X}_{j}$ and (d) the detected results. (e) The spatial distribution of the signed local shape distance overlaid on the fibular image forms the shape distance map.

surface of the patient's native mandible and that of the posttransfer fibular segments are first sampled at regular intervals, and the distance $d$ between the sampling points is calculated to determine local shape error. To calculate the mean of all sampling points on the fibular segment, the shape distance $E_{s}$ is estimated with the following formula:

$$
E_{s}=\frac{1}{n} \sum_{j=0}^{n-1} d\left(\boldsymbol{x}_{j}, \boldsymbol{X}_{j}\right)
$$

where $\boldsymbol{x}_{j}$ denotes a 3D sampling point on the implicit surface of the mandibular image $I$, and $\boldsymbol{X}_{j}$ is the corresponding sampling point on the surface of the fused fibular image $I_{f}^{\prime}$. The Euclidean distance between the two points is $d$, and $n$ is the number of the point pair $\left(\boldsymbol{x}_{j}, \boldsymbol{X}_{j}\right)$. The distribution of $d\left(\boldsymbol{x}_{j}, \boldsymbol{X}_{j}\right)$ for all sampling points on the fibular segments forms a shape distance map. Fig. 4 shows an example of a shape distance map and the outline of the computation algorithm. The following four steps describe the computation algorithm:

STEP 1 The two-dimensional contour lines of the native mandible are obtained by scanning slice images of the mandibular volume $I$ (Fig. 4a). The slice images are generated parallel to the mandibular tangent plane, and the $y$ axis is used for this first scan.

STEP 2 The contour lines are re-sampled along the curve, and the sampling points $\boldsymbol{x}_{j}$ and the normal vectors $\boldsymbol{n}_{j}$ are obtained. By expanding the points using $\boldsymbol{n}_{j}$, the start points $\boldsymbol{s}_{j}$ for the second scan are defined around the mandible (Fig. 4b).

STEP 3 The corresponding sampling point $\boldsymbol{X}_{j}$ of $\boldsymbol{x}_{j}$ is detected by scanning the oriented fibular image $I_{f}^{\prime}$ in the direction of $\boldsymbol{n}_{j}$ from $\boldsymbol{s}_{j}$. (Fig. 4c). If no corresponding point is found, the scan is terminated.

STEP 4 The point pair is obtained for all sampling points (Fig. 4d), and $E_{s}$ is calculated using (3).

To evaluate shape with sufficient accuracy, voxel resolution of the original CT images was used for a first sampling rate of the outer surface of the mandible in Step 1. If the axis-aligned axis (i.e. $y$ axis) is used for the second scan of $I_{f}^{\prime}$, the detected point pair $\left(\boldsymbol{x}_{j}, \boldsymbol{X}_{j}\right)$ is strongly affected by the posture of the mandible. To make the scan direction orientation-invariant and to place the sampling points as regularly as possible, we resampled the contours and used their normal vectors as the second scan directions to detect $\boldsymbol{X}_{j}$. The distribution of $d$ quantifies the local shape distance of the two implicit surfaces of $I$ and $I_{f}^{\prime}$. The red color overlaid on the fibular segments in left of Fig. 4e indicates that the section of the fibular implants near the connection point protrudes from the patient's native mandible. In contrast, blue regions indicate areas of depression from the patient's native mandible shown in right of Fig. 4e. Green indicates areas in which the local distance equals zero; that is, the placed fibular implants locally fit the patient's native mandible.

3) Symmetry distance: In this section, the mirror-symmetric distance $E_{r}$, which is the second shape descriptor, will be explained along with its method of calculation. This index is intended to quantify mandibular symmetry after fibular transfer surgery. The shape of the left and right mandible, i.e., the shape of the mandible in the regions where the $x$ coordinate has a positive versus negative value, are compared using the sagittal plane of the mandibular coordinate system as the plane of symmetry. A value of 0 is assigned if the left and right sections have complete mirror symmetry; increasing values indicate increasing asymmetry. To help define the mandibular 


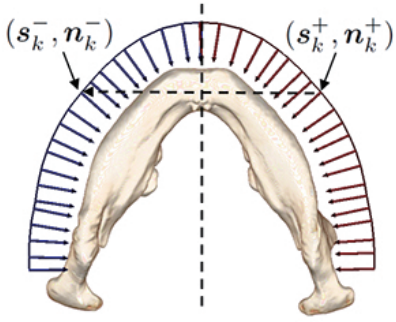

(a)

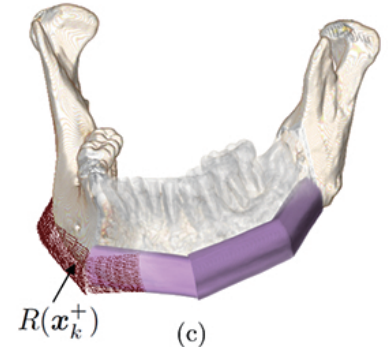

(c)

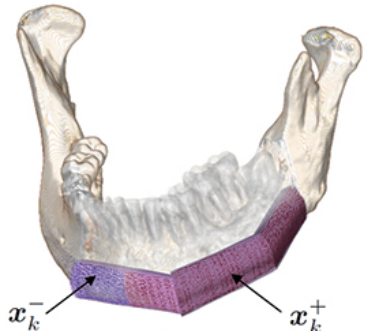

(b)

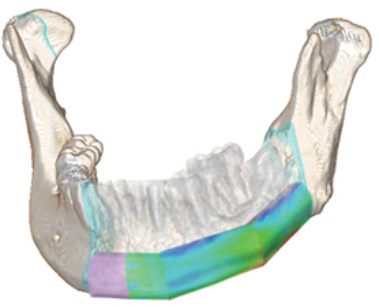

(d)
Fig. 5. Symmetric distance computation. (a) mirror-inverted scanning vectors placed around the mandible, (b) $\boldsymbol{x}_{k}^{-}$detected on the reconstruction plan, (c) $R\left(\boldsymbol{x}_{k}^{+}\right)$computed with symmetric transform, (d) spatial distribution of local symmetric distance.

shape after fibular segment transfer, the mandibular sagittal plane is used as the axis to mirror-invert the mandible in the region of $x>0$, and the shape distance between the mirrorinverted mandible and the mandible in the region of $x<0$ is calculated.

The outline of the calculation process is shown in Fig. 5. First, the surface positions $\boldsymbol{x}_{k}$ of the mandible and fibular segment are detected in a manner similar to that used for calculation of the shape distance, whereas, in this case, the scan is performed to the fused image of the mandible $I$ and the oriented fibula $I_{f}$. Of the detected $\boldsymbol{x}_{k}$, the position located at $x>0$ is defined as $\boldsymbol{x}_{k}^{+}$, and the normal vector of the average line corresponding to $\boldsymbol{x}_{k}^{+}$is defined as $\boldsymbol{n}_{k}^{+}$. The scanning starting point located on the outside of the mandible, which is used during detection of each $\boldsymbol{x}_{k}^{+}$, is defined as $\boldsymbol{s}_{k}^{+}$. Next, as shown in Fig. 5a, the mandibular sagittal plane is used as the plane of symmetry to mirror-invert $\boldsymbol{n}_{k}^{+}$and $\boldsymbol{s}_{k}^{+}$. The point $\boldsymbol{s}_{k}^{-}$ at which $s_{k}^{+}$is mirror-inverted, and the mirror-inverted vector $\boldsymbol{s}_{k}^{-}$of $\boldsymbol{n}_{k}^{+}$are found using (4) and (5), respectively, with the unit vector of the positive direction of the $x$ axis set as the normal vector of the plane of symmetry and defined as $\boldsymbol{n}$.

$$
\begin{array}{r}
\boldsymbol{n}_{k}^{-}=\boldsymbol{n}_{k}^{+}-2\left(\boldsymbol{n} \cdot \boldsymbol{n}_{k}^{+}\right) \boldsymbol{n} \\
\boldsymbol{s}_{k}^{-}=\boldsymbol{s}_{k}^{+}-2\left(\boldsymbol{n} \cdot \boldsymbol{s}_{k}^{+}\right) \boldsymbol{n}
\end{array}
$$

Next, as shown in Fig. 5b, the mandible is scanned from $\boldsymbol{s}_{k}^{-}$in the direction of $\boldsymbol{n}_{k}^{-}$to detect the surface position $\boldsymbol{x}_{k}^{-}$of the left mandible. $\boldsymbol{x}_{k}^{+}$is similarly found by applying mirror inversion, as shown in Fig. 5c. Point $R\left(\boldsymbol{x}_{k}^{+}\right)$is obtained following mirror inversion based on (6).

$$
R\left(\boldsymbol{x}_{k}^{+}\right)=\boldsymbol{x}_{k}^{+}-2\left(\boldsymbol{n} \cdot \boldsymbol{x}_{k}^{+}\right) \boldsymbol{n}
$$

Here $\boldsymbol{x}_{k}^{-}$is the point corresponding to $\boldsymbol{x}_{k}^{+}$on the left side of the mandible. The coordinates of $R\left(\boldsymbol{x}_{k}^{+}\right)$and $\boldsymbol{x}_{k}^{-}$coincide if the shape of the mandible is mirror-inverted; the distance between $R\left(\boldsymbol{x}_{k}^{+}\right)$and $\boldsymbol{x}_{k}^{-}$increases as the mandible becomes more asymmetrical. The formula $d\left(R\left(\boldsymbol{x}_{k}^{+}\right), \boldsymbol{x}_{k}^{-}\right)$is therefore taken as the local mirror-symmetric distance (see Fig. 5d), and the average of each sampling point is the mirror-symmetric distance $E_{r}$ defined by (7), where $m$ is the number of sampling points of the mandibular surface position $x_{k}^{-}$in the region of $x \leq 0$.

$$
E_{r}=\frac{1}{m} \sum_{k=0}^{m-1} d\left(R\left(\boldsymbol{x}_{k}^{+}\right), \boldsymbol{x}_{k}^{-}\right)
$$

Similar to $E_{s}$, a voxel interval was set for sampling of the outer edge of the mandibular surface in Step 1 to evaluate the shape with sufficient accuracy. An example of detection of the surface position $x_{k}^{-}$for $x<0$ is shown in Fig. $5 \mathrm{~b}$, while an example of calculation of the mirror-inverted mandibular surface position $R\left(\boldsymbol{x}_{k}^{+}\right)$for $x>0$ is shown in Fig. 5c.

STEP 1 Using the same procedure as that described in shape distance computation, the surface positions $\boldsymbol{x}_{k}$ of the mandible and fibular segment are identified.

STEP 2 Of the detected $\boldsymbol{x}_{k}$, the position located at $x>0$ is defined as $\boldsymbol{x}_{k}^{+}$. The normal vector corresponding to $\boldsymbol{x}_{k}^{+}$is defined as $\boldsymbol{n}_{k}^{+}$, whereas the scanning starting point is defined as $\boldsymbol{s}_{k}^{+}$.

STEP 3 With the $y z$ plane as the plane of symmetry, mirror-symmetric transformation is applied to $s_{k}^{+}$ and $\boldsymbol{n}_{k}^{+}$to obtain $\boldsymbol{s}_{k}^{-}$and $\boldsymbol{n}_{k}^{-}$.

STEP 4 The surface position $\boldsymbol{x}_{k}^{-}$of the mandible in the region of $x<0$ is detected from $s_{k}^{-}$in the direction of $\boldsymbol{n}_{k}^{-}$.

STEP $5 \boldsymbol{x}_{k}^{+}$is transformed to obtain the point $R\left(\boldsymbol{x}_{k}^{+}\right)$.

STEP 6 The distance between $\boldsymbol{x}_{k}^{-}$and $R\left(\boldsymbol{x}_{k}^{+}\right)$is calculated to obtain $d\left(R\left(\boldsymbol{x}_{k}^{+}\right), \boldsymbol{x}_{k}^{-}\right)$.

STEP 7 Steps $1-6$ are performed while changing the $z$ coordinates of the plane at regular intervals.

STEP 8 The average of $d\left(R\left(\boldsymbol{x}_{k}^{+}\right), \boldsymbol{x}_{k}^{-}\right)$, which is obtained in Steps 1-7, is calculated to give the mirrorsymmetric distance $E_{r}$ defined by the reconstruction parameter $\boldsymbol{\theta}$.

\section{Multivariate objective function design}

In this section, the design of the objective functions used in automation and their optimization methods are discussed. The objective function $E$ is designed to evaluate the reconstructed shape by connecting points based on the shape descriptors introduced above. Here, the sought reconstruction parameter, as shown in (2), represents the solution that minimizes $E$. The shape distance $E_{s}$ and mirror-symmetric distance $E_{r}$ are indices that quantify the postoperative shape of the mandible from different perspectives. Fig. 6 shows visualization results of the two indices for the two-segment procedures shown in Fig. 2. The upper image shows that $E_{s}$ is relatively small but $E_{r}$ is large, whereas the lower plan shows a large $E_{s}$ and 


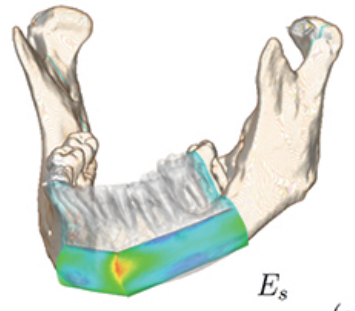

(a)
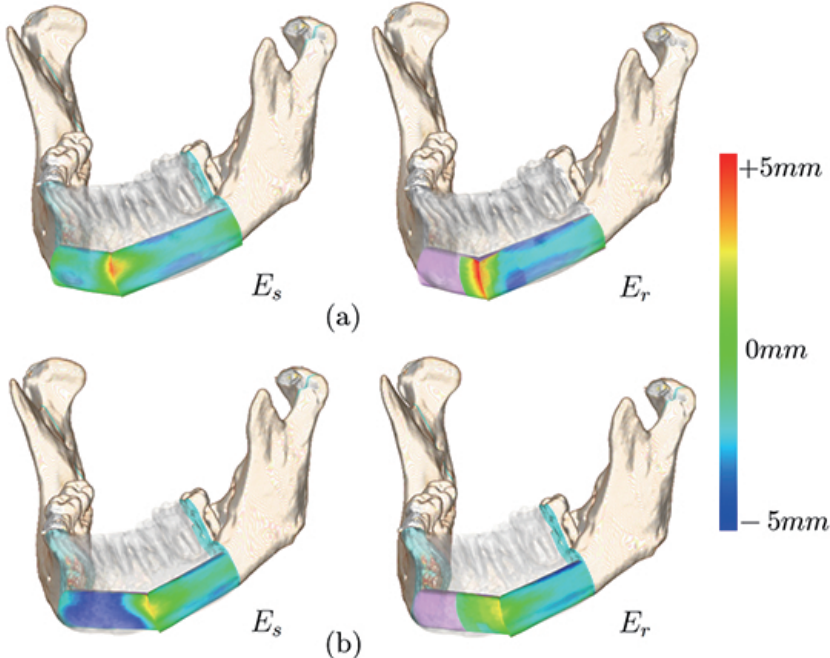

Fig. 6. Spatial distribution of the signed local shape distance and mirrorsymmetric distance. Two placement examples (a) with small $E_{s}$ and large $E_{r}$ and (b) with large $E_{s}$ and small $E_{r}$. The indices evaluate different geometrical aspects of the reconstruction plans.

small $E_{r}$. These differences demonstrate that reconstruction planning requires compromises because of the variety of 3D mandibular defects and the limited contour possibilities of fibular flaps.

Combining these two indices is expected to produce reconstruction results similar to those achieved with surgeondesigned plans. Moreover, while surgeons usually prefer placement that minimizes the shape error between the native mandible and the fibular segment, large local projections and depressions can occur with fibular segment placement that minimizes the average local shape error. Projecting fibular segments can be partially cut during surgery, but minimal additional cutting is preferable to preserve the strength of the bone graft. In other words, placement with a projection that is within a certain range is desirable. The amount of projection $E_{p}$ can be calculated with (8) and may be considered as the maximum value of the signed local shape distance.

$$
E_{p}=\max _{j \in[0, n-1]} \boldsymbol{n}_{j}\left(\boldsymbol{x}_{j}-\boldsymbol{X}_{j}\right)
$$

The objective function $E$ that quantifies the reconstructed shape is described as the weighted linear sum of the shape distance $E_{s}$ and the mirror-symmetric distance $E_{r}$ and is written as (9).

$$
E(\lambda)=E_{s}+\lambda E_{r}
$$

Constraints are also introduced for the maximum value $E_{p}$ of the signed local shape error. In other words, the reconstruction planning process is formulated as a constrained minimization problem, as shown in (10).

$$
\boldsymbol{\theta}^{*}=\underset{\boldsymbol{\theta}}{\operatorname{argmin}} E(\lambda) \quad \text { subject to } E_{p} \leq t h
$$

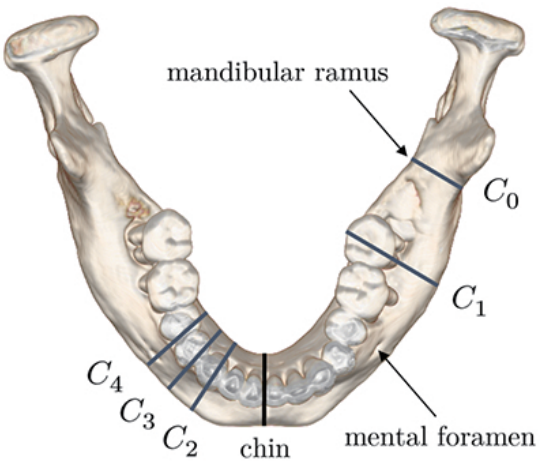

Fig. 7. Five cutting planes based on anatomical features of the mandible.

It is not evident here which index should be focused on and to what degree, in case of different resection areas or mandibular shapes with individual differences. Therefore, $\lambda$ is determined through experiments using case CT data. Equation (10) can be described as (11).

$$
\begin{gathered}
\boldsymbol{\theta}^{*}=\underset{\boldsymbol{\theta}}{\operatorname{argmin}}\left[\frac{1}{n} \sum_{j=0}^{n-1} d\left(\boldsymbol{x}_{j}, \boldsymbol{X}_{j}\right)+\frac{\lambda}{m} \sum_{k=0}^{m-1} d\left(R\left(\boldsymbol{x}_{k}^{+}\right), \boldsymbol{x}_{k}^{-}\right)\right] \\
\text {subject to } \max _{j \in[0, n-1]} \boldsymbol{n}_{j}\left(\boldsymbol{x}_{j}-\boldsymbol{X}_{j}\right) \leq t h
\end{gathered}
$$

When deriving the connection points that minimize the objective function $E$, a full search in which all scores within a certain area are found while the position of the connection point is moved at fixed step widths can require an enormous amount of computation time. For example, even in the case of $N=2$, moving the connection point at step widths of $1 \mathrm{~mm}$ over an area of $30 \mathrm{~mm} \times 30 \mathrm{~mm} \times 30 \mathrm{~mm}$ takes approximately $45 \mathrm{~min}$ with current general-purpose computers. With three segments, calculations cannot be completed within a reasonable time, because the number of parameters doubles.

The coordinates of the connection points that minimize nonconvex objective function $E$ are found using the Covariance Matrix Adaptation Evolution Strategy (CMA-ES) [44]. The CMA-ES consists of a series of steps, including sampling point acquisition, selection and update of the distribution center, update of the step size, and update of the covariance matrix. A trial of this optimization process on several variations of these parameters produced a sampling number (i.e., a population size) of six, considering the number of iterations required for convergence and the stability. A parameter of $10 \mathrm{~mm}$ was set as the standard deviation used to determine the sampling area at each iteration.

\section{EXPERIMENTS AND RESULTS}

Experiments were performed to evaluate the automated fibular transfer planning using the designed objective functions. The planning results were evaluated by investigating whether the automated planning results approximated the manual reconstruction plan based on the surgeon's knowledge and experience. The efficacy of the multivariate objective 


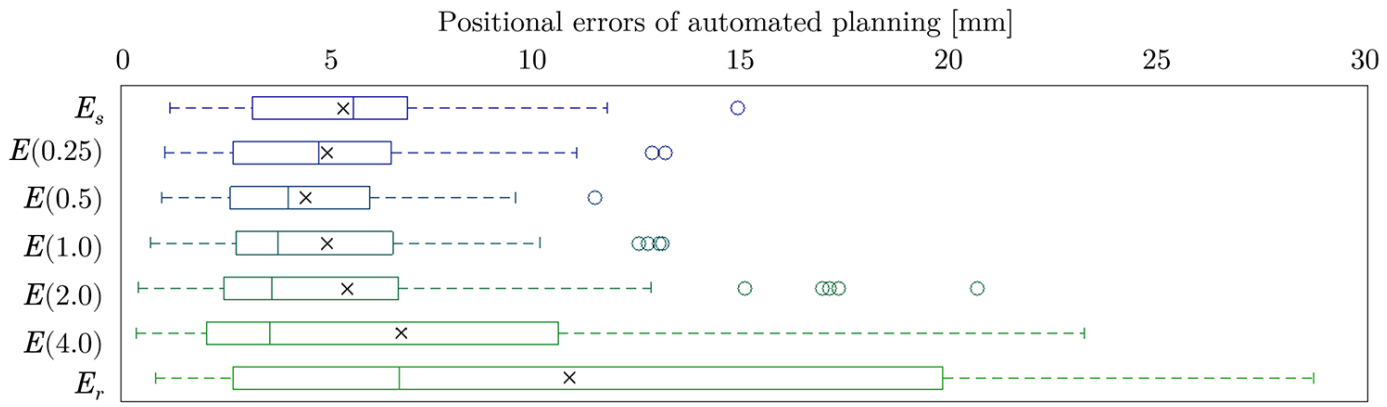

(a)

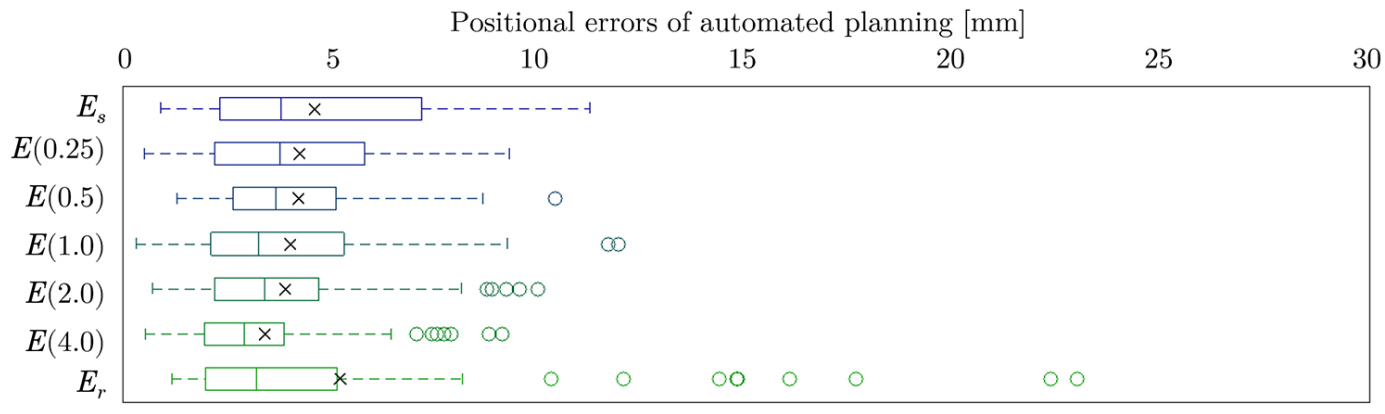

(b)

Fig. 8. Positional errors of the automated plans for (a) $N=2$ and (b) $N=3$. The box plots include the minimum, first quartile $(Q 1)$, median $(Q 2)$, third quartile $(Q 3)$ and maximum. The minimum and maximum scores are represented after outliers have been rejected. Values larger than $(Q 3-Q 1) \times 1.5+Q 3$ or smaller than $Q 1-(Q 3-Q 1) \times 1.5$ were regarded as outliers. The mean scores are indicated by plotted $\times$ 's.

function was also confirmed by comparing it with existing planning models. Four medical staff (one oral surgeon and two dental technicians at Nara Medical University, and one oral surgeon at Rakuwakai Otowa Hospital) participated in the experiments.

\section{A. Acquisition of reconstruction plans}

Ten CT datasets (Case1-10) of both the head and lower leg were used for this evaluation. The datasets included geometrical variations of patients' native mandibles, as shown in Fig. 2. The CT slice images were first re-sampled as regularized volume data with $256^{3}$ voxels. The mandible region $I$ was extracted from the head CT data, and the fibular image $I_{f}$ was extracted from the lower leg CT data. This segmentation step was completed in 1-3 min by selecting the region of interest then manually removing voxels with a $3 \mathrm{D}$ cutting tool [45]. To retrospectively reproduce the preoperative planning whilst maintaining reproducibility, we defined five cutting planes, based on the anatomical feature points of the mandible: $C_{k}(k=0, \ldots, 4)$. The rotations of the cutting planes are uniquely determined because they are perpendicular to the outline of the mandibular contour.

- $C_{0}$ : mandibular ramus

- $C_{1}$ : midpoint of $C_{0}$ and $C_{4}$

- $C_{2}$ : midpoint of chin and $C_{4}$

- $C_{3}$ : midpoint of $C_{2}$ and $C_{4}$

- $C_{4}$ : mental foramen

Six patterns of the asymmetric cutting region: $\left(C_{0}, C_{2}\right)$, $\left(C_{0}, C_{3}\right),\left(C_{0}, C_{4}\right),\left(C_{1}, C_{2}\right),\left(C_{1}, C_{3}\right)$, and $\left(C_{1}, C_{4}\right)$; were then defined from a combination of the cutting planes (see
Fig. 7). We confirmed that these cutting patterns were similar to those used in past surgeries.

The surgeons and technicians (hereafter termed participants) reproduced the surgical planes using interactive planning software [41]. During this experiment, two types of mandibular reconstruction, using two or three fibular segments $(N=2$ or 3), were simulated. Their current states were displayed on two monitors, both with the same specifications (i.e., same window size and same resolution). The participants watched both displays and determined the most suitable positions for the connection points and angles of the virtual surfaces. When all participants agreed with the planning results, the reconstruction plan was fixed and recorded. Sixty reconstruction patterns (ten CT datasets and six types of cutting regions) were presented, and 120 planning results on two- and three-segment procedures were obtained.

\section{B. Experimental methods}

We computed the planning results with the objective function $E(\lambda)$, using 60 resection area patterns, to investigate the effect of the parameter $\lambda$. The optimum value for $\lambda$, which produced the solution closest to the manual placement by the surgeon, was determined from six values: $0.0,0.25,0.5$, 1.0, 2.0 and 4.0, prepared as typical weight parameters. After determining the optimal value of $\lambda$ for two- and three-segment procedures, the proposed planning models were compared with one existing reconstruction model based on the following objective functions.

1) $E$ : multivariate objective function defined by (9). 


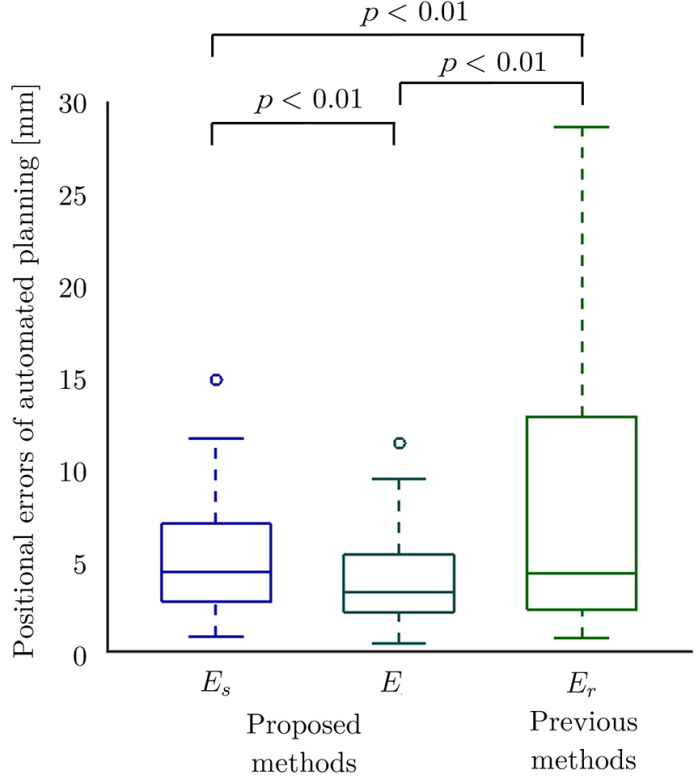

Fig. 9. Box plots and ANOVA test results of positional errors for 120 automated plans. Statistical significance was observed in all pairs of the three models.

2) $E_{s}$ : objective function using shape distance described by (3), which is a generalized automatic planning model of [14].

3) $E_{r}$ : objective function using symmetric distance defined by (7). This model is a possible automatic planning based on a previous mirror-image-based reconstruction [28], [39], [40].

Throughout the experiments, the initial connection point $\boldsymbol{p}_{k}$ was defined as the midpoint uniquely calculated by linear interpolation from the connection points $\boldsymbol{p}_{0}$ and $\boldsymbol{p}_{N}$ at both ends. A sampling number of six and a standard deviation of 10 $\mathrm{mm}$ were used for the parameters of the CMA-ES. The CMAES was performed with 30 iterations, which were enough interactions for convergence; in this case. $E_{p}$ was calculated from the manual planning results and its median value of 5.67 $\mathrm{mm}$ was used as $t h$ in (10).

\section{Automated planning results}

To quantitatively evaluate the automated planning results, we measured the positional error between the connection points of the automated plans and those of the surgeon. The positional error in $N=2$ was computed using the Euclidean distance of the corresponding connection points $\boldsymbol{p}_{1}$, obtained from automatic and manual placement. The positional error in $N=3$ was computed as the mean positional errors of $\boldsymbol{p}_{1}$ and $p_{2}$.

Fig. 8 shows the box plots of the positional errors of the automated plans with two fibular segments $(N=2)$ and three fibular segments $(N=3)$. The mean positional errors for the two-segment procedure were $5.36 \mathrm{~mm}$ and $10.82 \mathrm{~mm}$, in $E_{s}$ and $E_{r}$, respectively. The smallest error was $4.4 \mathrm{~mm}$, in the case of $E(0.5)$. The greater the $\lambda$ score deviated from 0.5 , the
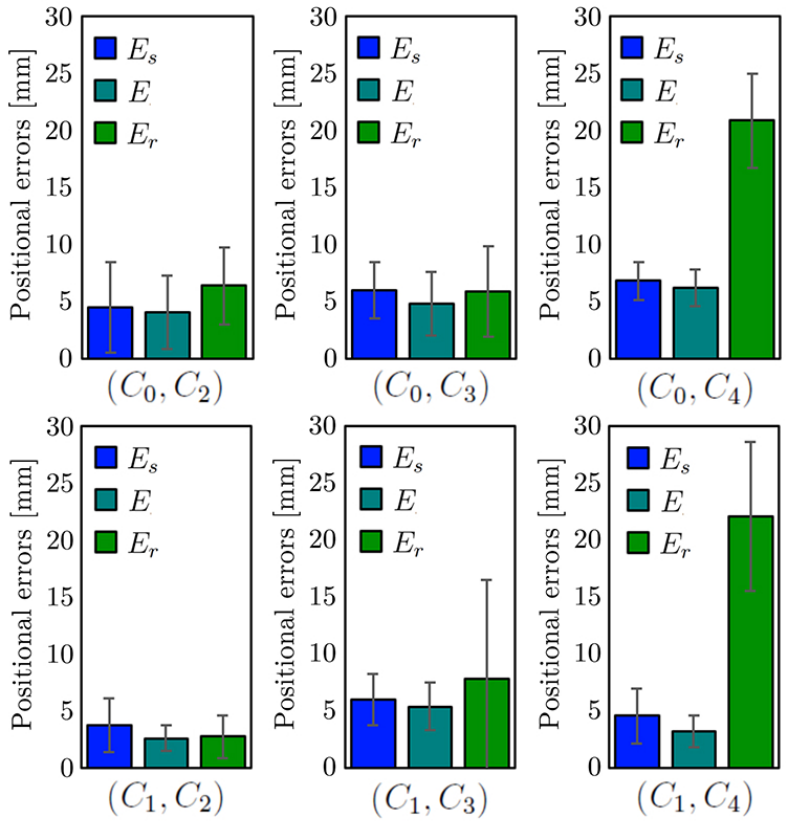

(a)
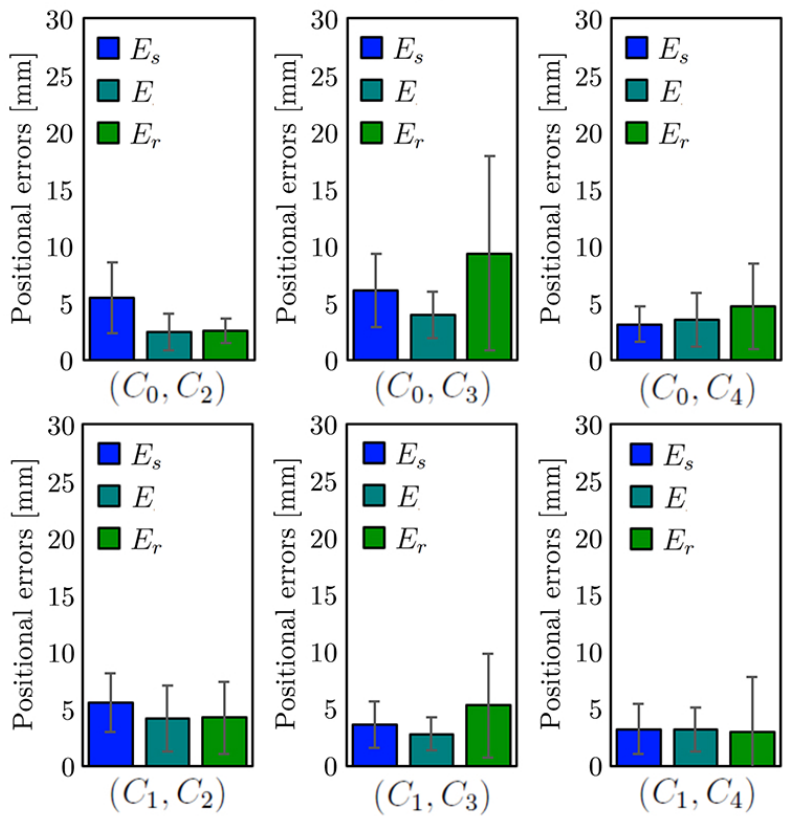

(b)

Fig. 10. The positional errors of the automated plans for six patterns of resection areas. (a) two-segment procedures and (b) three-segment procedures.

greater the positional error became. The mean positional errors for the three-segment procedure were $4.6 \mathrm{~mm}$ and $5.2 \mathrm{~mm}$, in $E_{s}$ and $E_{r}$, respectively. The smallest mean positional error for $N=3$ was $3.4 \mathrm{~mm}$, in the case of $E(4.0)$. The histogram of the positional error differs from those of the two-segment procedures. Overall, the shape of the boxplot became more similar to that of $E_{s}$ as $\lambda$ became smaller, and more similar to $E_{r}$ as $\lambda$ became larger. This is consistent with the fact that $E_{s}$ is equivalent to $\lambda=0$ in $E$, and $E_{r}$ is equivalent to $\lambda \rightarrow \infty$ in $E$. Based on the results, we determined that 0.5 and 


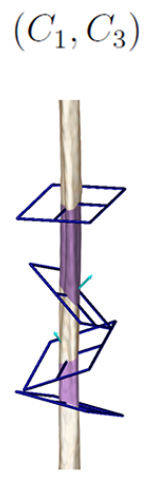

Positional error

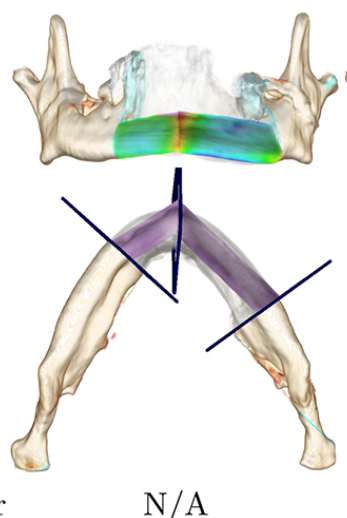

$\mathrm{N} / \mathrm{A}$

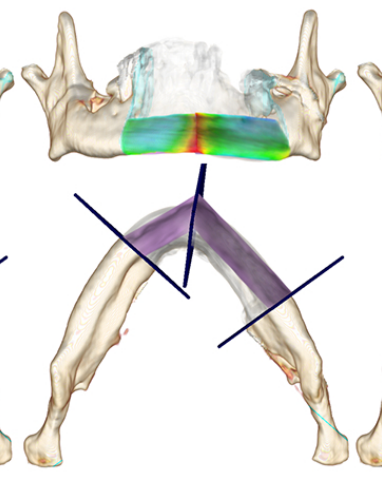

$3.43 \mathrm{~mm}$

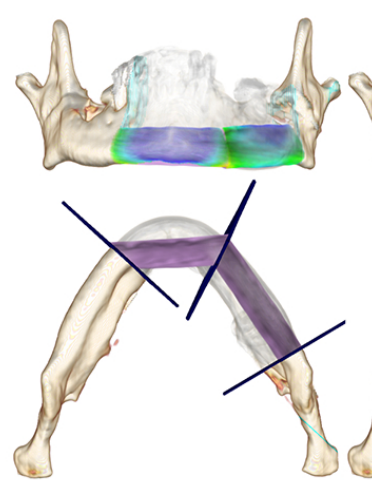

$\mathrm{N} / \mathrm{A}$

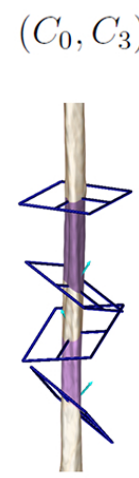

Positional error

E

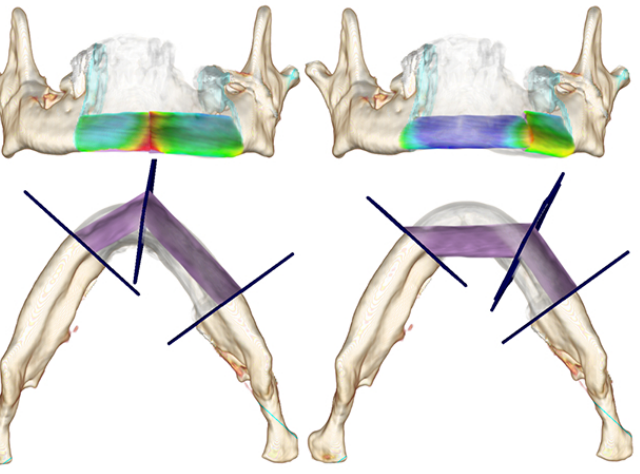

$3.53 \mathrm{~mm}$
$23.82 \mathrm{~mm}$ Surgeons' plan

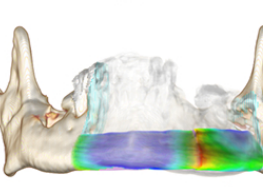

$E_{s}$

$E_{r}$

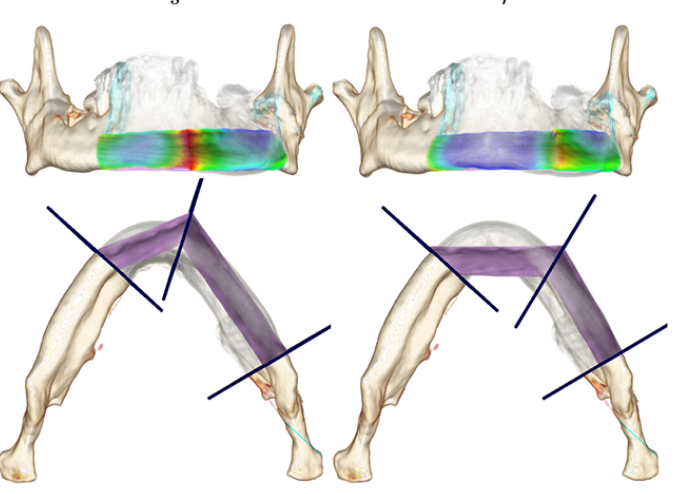

$11.7 \mathrm{~mm}$

$6.21 \mathrm{~mm}$

\begin{abstract}
$3.93 \mathrm{~mm}$
\end{abstract}
E

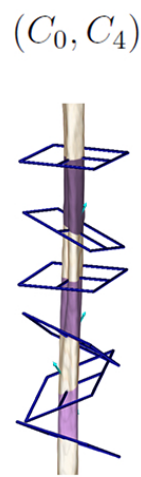

Positional error

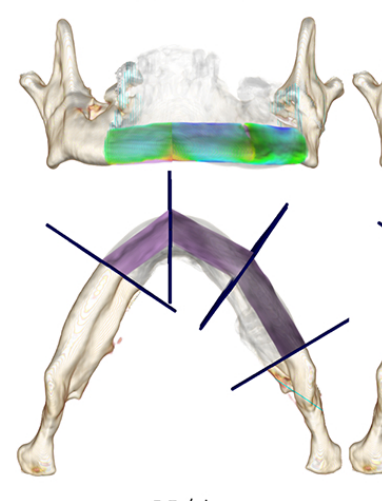

$\mathrm{N} / \mathrm{A}$

Surgeons' plan

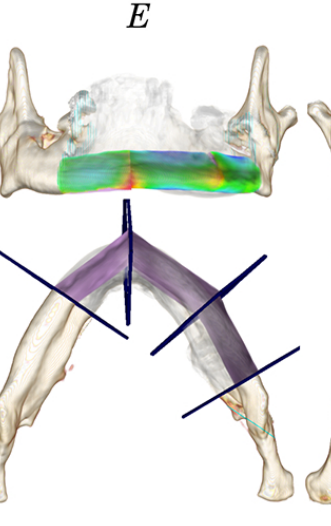

$2.76 \mathrm{~mm}$

E
$E_{s}$

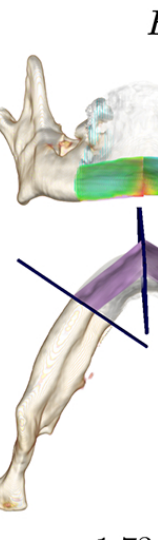

$1.73 \mathrm{~mm}$

$E_{s}$
$E_{r}$

E

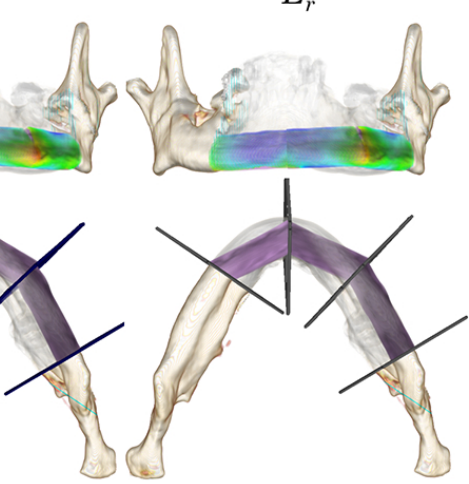

$4.39 \mathrm{~mm}$

$E_{r}$

Fig. 11. Illustrative examples of the surgeons' plans and the typical automated plans, where $E_{s}, E$ and $E_{r}$ were used as objective functions.

4.0 were the optimal values of $\lambda$ for two- and three-segment procedures, respectively.

Fig. 9 shows the box plots of the positional error for 120 planning results when the proposed planning models with $E_{s}$ and $E$, and the existing planning model with $E_{r}$, were applied. Fig. 9 also includes ANOVA test results between each pair of the three models; statistical significance was observed in all pairs. The proposed planning models with $E$ and $E_{s}$ had a smaller mean positional error and showed less variation than the model with the function $E_{r}$. The multivariate mode with the function $E$ was better than that with $E_{s}$ for evaluating a single feature of the reconstruction plan.

Fig. 10 shows the mean positional errors and the standard deviation of the automated plans for six patterns of cutting regions. The automated plans with the objective function $E$ resulted in the smallest positional error in most parts of the cutting patterns. The positional errors of $E_{r}$ were, in some cases, extremely high for the $\left(C_{0}, C_{4}\right)$ and the $\left(C_{1}, C_{4}\right)$ regions in two-segment procedures, and relatively high for large cutting regions such as in $\left(C_{0}, C_{3}\right)$ and $\left(C_{1}, C_{3}\right)$.

Fig. 11 shows illustrative examples of the typical automated planning results; two-segment procedures for the $\left(C_{1}, C_{3}\right)$ 
and $\left(C_{0}, C_{3}\right)$ regions and three-segment procedures for the $\left(C_{0}, C 4\right)$ regions in Case 1 , where $E_{s}, E$ and $E_{r}$ were used as objective functions. The upper images in each situation show the shape distance map, which visualizes the local shape distance of the mandible and the grafted fibular segments. The surgeons' plan is shown in the left column and the positional errors of each automated plan are denoted below each row of figure sets. The leftmost figures are the osteotomy lines for fibular shaping obtained from the automated plans by $E$.

For the $\left(C_{1}, C_{3}\right)$ region, the reconstruction plans using $E$ and $E_{s}$ were similar to those of the surgeon, whereas the plan using $E_{r}$ was different. The positional errors for $E, E_{s}$ and $E_{r}$ were $3.43 \mathrm{~mm}, 3.53 \mathrm{~mm}$ and $23.82 \mathrm{~mm}$, respectively. Since $E_{r}$ attempts to achieve mandibular symmetry in postreconstruction by minimizing the symmetric distance, the reconstruction plan using $E_{r}$ has resulted in a larger depression in the mental region of the mandible, and the positional error is high. In contrast, for the $\left(C_{0}, C_{3}\right)$ region, $E$ and $E_{r}$ were close to the surgeons' plan. The reconstruction plan using $E_{s}$ showed a high positional error. The positional errors for $E, E_{s}$ and $E_{r}$ were $3.93 \mathrm{~mm}, 11.7 \mathrm{~mm}$ and $6.21 \mathrm{~mm}$, respectively. Although the surgeons' plan in this region considers the degree of symmetry in the post-operative mandibular arc, $E_{s}$ fails to capture this factor because it concentrates on the minimization of the average shape difference between the preoperative native mandible and the postoperative grafted fibula. Similar cases were found in three-segment procedures (the lowest row of images in Fig. 11). The reconstruction plan using $E_{r}$ yields a depression around the chin. The positional error of $E_{s}$ was slightly smaller than that of $E$ in this case, but based on the surgeons' comment, $E$ provides a good reconstruction plan in terms of both degree of symmetry and the contour of the native mandible.

Fig. 12 shows the 2D distribution of each score for the $\left(C_{1}, C_{3}\right),\left(C_{0}, C_{3}\right)$ region in Case 1 , when the position of the connection point was updated per $1 \mathrm{~mm}$ pitch in the $x$ and $y$ directions in the mandibular tangent plane. The image orientation is the same as that in Fig. 3b. The center of the image is the location of the connection point manually placed by the surgeon. In the upper images, scores decrease near the center of the color maps for $E$ and $E_{s}$, whereas for $E_{r}$, the distribution of low scores is located in the lower right in the color map. In the lower images for the $\left(C_{0}, C_{3}\right)$ region, the distribution of the low scores in $E_{s}$ differs greatly from that in $E_{r}$.

We measured the computation time required for the CMAES based optimization process in two- and three-segment procedures of 60 cutting patterns. A computer with graphic processing units (CPU: Intel Corei7 3.5GHz, Memory: $8 \mathrm{~GB}$, GPU: NVIDIA GeForce 780) was used for this measurement. The average computation time and the standard deviation were $33.3 \pm 3.8 \mathrm{sec}$ for two-segment procedures and $43.4 \pm 5.5 \mathrm{sec}$ for three-segment procedures, which demonstrates clinically reasonable performance for preoperative planning use. GPUbased parallel computation of the shape descriptors was effective in achieving this fast computation.

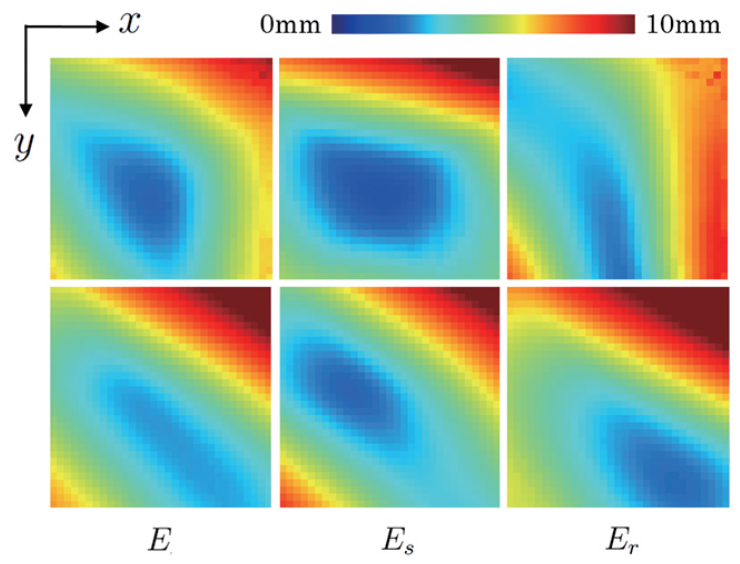

Fig. 12. 2D distribution of scores using different objective functions.

\section{Discussion}

In this paper, we have introduced automated planning methods for fibular transfer surgery in mandibular reconstruction. The performance of the proposed methods, using the multivariate objective function $E$, was compared with a previous planning model. Fig. 9 showed that optimization with a single feature (the shape distance or the degree of symmetry alone) could fail to reproduce the surgeons' reconstruction plans. This instability is observed in the variation and long tails with outliers, as shown in Fig. 8. A possible reason for this variation could be the incompatible placement of just one of the indices. Fig. 12 also reveals that the symmetric reconstruction and restoration of the native contour of the mandible include tradeoff problems, and this should be carefully considered, especially in two-segment reconstruction cases for large cutting regions.

The oral surgeons who participated in the experiments commented that decision making on this trade-off problem is usually subjectively made based on the estimated visual appearance and surgeons' knowledge and experience. This is done even when a commercial product or interactive virtual planning software is being used. The study addressed such quantitatively unclear issue, and made preoperative planning more systematic. The shape difference, with respect to the patient's native mandible, and postoperative mandibular symmetry were quantified using the two descriptors: $E_{s}$ and $E_{r}$, respectively. The experimental results suggest that a weighted linear combination of the two indices could prevent the extreme deviations in placement that occur when either of the indices is used alone. These findings showed automated planning with multivariate objective function could formulate the decision making of geometrical aspects. Multivariate factor analysis that does not depend on individual subjectivity has potential clinical benefit for systematic, quantitative understanding of surgical procedures in addition to patient-specific preoperative planning.

One limitation of this study was that the automated planning framework was designed in terms of optimizing shape restoration of the mandible. The current methods suppose that full geometry of native mandible is obtained before surgery, 
and cases with prior defects or malformations of mandibles are not considered. For these cases, use of statistical shape models (SSM) [22] can be potentially a good solution. The use of partially known geometries for estimating the missing parts was investigated in medical image analysis research [38]. The concept of SSM requires a large dataset to achieve clinically valid shape estimation. However, if SSMs are available, the objective function may provide reasonable planning results that minimize the difference between the SSM and the posttransfer shape even if the whole shape of the native mandible is not available. As the presented planning framework is compatible with SSMs, their interaction will be an interesting research topic that may increase their clinical value.

From clinical aspects, as mentioned in Section II, many issues that influence the reconstruction should be considered to improve clinical applicability of the overall plan. The vascular pedicle and the shape of the soft-tissue flap transplanted with fibula were modeled in past studies [33], [34]. The consideration of malocclusion, biomechanical or physiological aspects of the mandibular reconstruction [32] is important for restoration of mastication function. Additional parameters at the harvesting site can be considered to formulate the procedures of osteotomy. Integrating these factors with automated planning would further improve clinical values of the overall software framework.

The proposed methods are potentially applicable to other reconstructions such as upper jaw reconstruction [36], more complex placement of fibula flaps [12] and skull reconstruction [19]. For further improvement of the method, fully automatic coordination or parameterization of the mandible could be investigated. The objective function could be optimized for the detailed resection patterns. Exploration of effective planning features and the fine tuning of the planning parameters will be an interesting topic, leading to the development of a statistical atlas or quantified classification of oncological resection in mandibular reconstruction. Multi-facility research, including the comparison of planning parameters between hospitals, will be important for procedure standardization.

\section{CONCLUSION}

The aim of this study was to automate the process of determining fibular segmentation and placement during preoperative planning for mandibular reconstruction using fibular flaps, a process that previously required numerous manual parametric adjustments and decisions based on a surgeon's knowledge and experience. Shape distance and mirror-symmetric distance were introduced as shape descriptors to multilaterally quantify fibular segment transfer to the patient-specific mandibular defects.

The efficacy of the proposed objective functions was verified by quantitatively comparing the automated plans with both the existing planning model and the surgeons' plans. The developed framework addressed trade-off problems between the symmetric reconstruction and restoration of the native contour of the mandible, and achieved stable automated planning that was similar to the surgeons' decision making. Our future work will be the further exploration of effective features to improve the stability of the automated planning, and the development of a statistical atlas for the classification of mandibular reconstruction.

\section{ACKNOWLEDGEMENT}

This research was supported by JSPS Grant-in-Aid for Scientific Research (B) (15H03032) and the Center of Innovation (COI) Program of the Japan Science and Technology Agency (JST). We would like to thank Keiho Imanishi (eGrowth co.ltd.) for participating in a valuable discussion on the algorithm design and software implementation.

\section{REFERENCES}

[1] A. F. S. Flemming et al., "Mandibular reconstruction using vascularized fibula," British Journal of Plastic Surgery, vol. 43, no. 4, pp. 403-409, 1990.

[2] Y. Yamanaka et al., "Mandibular reconstruction with vascularised fibular osteocutaneous flaps using prefabricated stereolithographic mandibular model," Journal of Plastic Reconstructive and Aesthetic Surgery, vol. 63, no. 10, pp. 1751-1753, 2010.

[3] R. D. Foster et al., "Vascularized bone flaps versus nonvascularized bone grafts for mandibular reconstruction: An outcome analysis of primary bony union and endosseous implant success," Head and Neck, vol. 21, no. 1 , pp. 66-71, 1999.

[4] A. K. Antony et al., "Use of virtual surgery and stereolithography-guided osteotomy for mandibular reconstruction with the free fibula," Plastic and Reconstructive Surgery, vol. 128, no. 5, pp. 1080-1084, 2011.

[5] N. Ayoub et al., "Evaluation of computer-assisted mandibular reconstruction with vascularized iliac crest bone graft compared to conventional surgery: a randomized prospective clinical trial," Trials, vol. 15, p. 114,2014

[6] K. Smolka et al., "Fibula free flap reconstruction of the mandible in cancer patients: evaluation of a combined surgical and prosthodontic treatment concept," Oral Oncol, vol. 44, no. 6, pp. 571-581, 2008.

[7] D. L. Hirsch et al., "Use of computer-aided design and computer-aided manufacturing to produce orthognathically ideal surgical outcomes: A paradigm shift in head and neck reconstruction," Journal of Oral and Maxillofacial Surgery, vol. 67, no. 10, pp. 2115-2122, 2009.

[8] P. Juergens et al., "Computer simulation and rapid prototyping for the reconstruction of the mandible," Journal of Oral and Maxillofacial Surgery, vol. 67, no. 10, pp. 2167-2170, 2009.

[9] J. Sink et al., "Computer-aided stereolithography for presurgical planning in fibula free tissue reconstruction of the mandible," Journal of Reconstructive Microsurgery, vol. 28, no. 6, pp. 395-403, 2012.

[10] O. Tepper et al., "The new age of three-dimensional virtual surgical planning in reconstructive plastic surgery," Plastic and Reconstructive Surgery, vol. 130, no. 1, pp. 192e-194e, 2012.

[11] W. H. Wang et al., "Preoperative three-dimensional reconstruction in vascularized fibular flap transfer," Journal of Cranio-Maxillofacial Surgery, vol. 40, no. 7, pp. 599-603, 2012.

[12] W. H. Wang et al., "Three-dimensional virtual technology in reconstruction of mandibular defect including condyle using double-barrel vascularized fibula flap," Journal of Cranio-Maxillofacial Surgery, vol. 41, no. 5, pp. 417-422, 2013.

[13] M. Nakao et al., "Volumetric surgical planning system for fibular transfer in mandibular reconstruction," International Conference of the IEEE Engineering in Medicine and Biology Society, pp. 3367-3370, 2013.

[14] M. Nakao et al., "Volumetric fibular transfer planning with shape-based indicators in mandibular reconstruction," IEEE Journal of Biomedical and Health Informatics, vol. 19, no. 2, pp. 581-589, 2015.

[15] N. Kozic et al., "Optimisation of orthopaedic implant design using statistical shape space analysis based on level sets," Med Image Anal, vol. 14, no. 3, pp. 265-275, 2010.

[16] R. Blanc et al., "Statistical model based shape prediction from a combination of direct observations and various surrogates: application to orthopaedic research," Med Image Anal, vol. 16, no. 6, pp. 1156-1166, 2012.

[17] S. S. Chandra et al., "Focused shape models for hip joint segmentation in 3d magnetic resonance images," Med Image Anal, vol. 18, no. 3, pp $567-578,2014$ 
[18] M. E. de Oliveira et al., "Feature-invariant image registration method for quantification of surgical outcomes in patients with craniosynostosis: a preliminary study," J Pediatr Surg, vol. 46, no. 10, pp. E1-8, 2011.

[19] C. S. Mendoza et al., "Personalized assessment of craniosynostosis via statistical shape modeling," Med Image Anal, vol. 18, no. 4, pp. 635646, 2014.

[20] I. Otomaru et al., "Automated preoperative planning of femoral stem in total hip arthroplasty from $3 \mathrm{~d}$ ct data: atlas-based approach and comparative study," Med Image Anal, vol. 16, no. 2, pp. 415-426, 2012.

[21] M. L. Urken et al., "Oromandibular reconstruction using microvascular composite flaps: report of 210 cases," Arch Otolaryngol Head Neck Surg, vol. 124, no. 1, pp. 46-55, 1998.

[22] C. Seiler, X. Pennec, and M. Reyes, "Capturing the multiscale anatomical shape variability with polyaffine transformation trees," Med Image Anal, vol. 16, no. 7, pp. 1371-1384, 2012.

[23] I. Otomaru et al., "Automated preoperative planning of femoral component for total hip arthroplasty (tha) from $3 \mathrm{~d}$ ct images," Proceedings of Medical Imaging and Augmented Reality, vol. 5128, pp. 40-49, 2008.

[24] D. M. Steinbacher, "Three-dimensional analysis and surgical planning in craniomaxillofacial surgery," J Oral Maxillofac Surg, vol. 73, no. 12 Suppl, pp. S40-56, 2015.

[25] A. Tarsitano et al., "Mandibular reconstructions using computer-aided design/computer-aided manufacturing: A systematic review of a defectbased reconstructive algorithm," J Craniomaxillofac Surg, vol. 43, no. 9, pp. 1785-1791, 2015.

[26] D. Rohner et al., "Importance of patient-specific intraoperative guides in complex maxillofacial reconstruction," Journal of Cranio-Maxillofacial Surgery, vol. 41, no. 5, pp. 382-390, 2013.

[27] L. Ciocca et al., "Accuracy of fibular sectioning and insertion into a rapid-prototyped bone plate, for mandibular reconstruction using cadcam technology," J Craniomaxillofac Surg, vol. 43, no. 1, pp. 28-33, 2015.

[28] G. A. Khalifa et al., "Segmental mirroring: Does it eliminate the need for intraoperative readjustment of the virtually pre-bent reconstruction plates and is it economically valuable?" J Oral Maxillofac Surg, vol. 74 no. 3, pp. 621-630, 2015.

[29] S. M. Roser et al., "The accuracy of virtual surgical planning in free fibula mandibular reconstruction: Comparison of planned and final results," Journal of Oral and Maxillofacial Surgery, vol. 68, no. 11, pp. 2824-2832, 2010

[30] Y. Shen et al., "Using computer simulation and stereomodel for accurate mandibular reconstruction with vascularized iliac crest flap," Oral Surgery Oral Medicine Oral Pathology Oral Radiology, vol. 114, no. 2, pp. 175-182, 2012.

[31] B. D. Foley et al., "Mandibular reconstruction using computer-aided design and computer-aided manufacturing: An analysis of surgical results," Journal of Oral and Maxillofacial Surgery, vol. 71, no. 2, pp. E111-E119, 2013.

[32] J. Kimsal et al., "Biomechanical analysis of mandibular angle fractures," Journal of Oral and Maxillofacial Surgery, vol. 69, no. 12, pp. 30103014,2011

[33] L. Zhao, P. K. Patel, and M. Cohen, "Application of virtual surgical planning with computer assisted design and manufacturing technology to cranio-maxillofacial surgery," Archives of Plastic Surgery, vol. 39 , no. 4, pp. 309-316, 2012

[34] P. Olsson et al., "Haptics-assisted virtual planning of bone, soft tissue, and vessels in fibula osteocutaneous free flaps," Plast Reconstr Surg Glob Open, vol. 3, no. 8, p. e479, 2015.

[35] D. F. Zweifel et al., "Are virtual planning and guided surgery for head and neck reconstruction economically viable?" J Oral Maxillofac Surg, vol. 73, no. 1, pp. 170-175, 2015.

[36] R. J. Murphy et al., "Optimizing hybrid occlusion in face-jaw-teeth transplantation: A preliminary assessment of real-time cephalometry as part of the computer-assisted planning and execution workstation for craniomaxillofacial surgery," Plast Reconstr Surg, vol. 136, no. 2, pp. $350-362,2015$

[37] J. S. Brown et al., "A new classification for mandibular defects after oncological resection," The Lancet Oncology, vol. 17, no. 1, pp. e23e30, 2016

[38] S. Raith et al., "Planning of mandibular reconstructions based on statistical shape models," International Journal of Computer Assisted Radiology and Surgery, pp. 1-14, 2016.

[39] S. Kumta et al., "A novel 3d template for mandible and maxilla reconstruction: Rapid prototyping using stereolithography," Indian Journal of Plastic Surgery : Official Publication of the Association of Plastic Surgeons of India, vol. 48, no. 3, pp. 263-273, 2015.
[40] J. Damstra et al., "Combined 3-dimensional and mirror-image analysis for the diagnosis of asymmetry," Am J Orthod Dentofacial Orthop, vol. 140, no. 6, pp. 886-894, 2011.

[41] M. Nakao et al., "Statistical analysis of interactive surgical planning using shape descriptors in mandibular reconstruction with fibular segments," PLoS One, vol. 11, no. 9, p. e0161524, 2016.

[42] M. Nakao and K. Minato, "Physics-based interactive volume manipulation for sharing surgical process," IEEE Transactions on Information Technology in Biomedicine, vol. 14, no. 3, pp. 809-816, 2010.

[43] K. W. C. Hung et al., "Background-incorporated volumetric model for patient-specific surgical simulation: a segmentation-free, modeling-free framework," International Journal of Computer Assisted Radiology and Surgery, vol. 6, no. 1, pp. 35-45, 2011.

[44] N. Hansen and A. Ostermeier, "Completely derandomized selfadaptation in evolution strategies," Evol Comput, vol. 9, no. 2, pp. 15995, 2001.

[45] K. Imanishi et al., "Interactive bone drilling using a $2 \mathrm{~d}$ pointing device to support microendoscopic discectomy planning," International Journal of Computer Assisted Radiology and Surgery, vol. 5, no. 5, pp. 461-469, 2010.

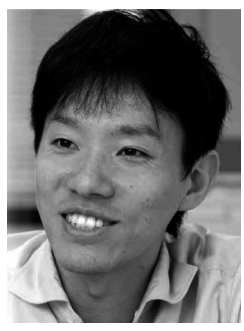

Megumi Nakao received the Ph.D. degree in informatics from Kyoto University, Kyoto, Japan, in 2003. After working at Graduate School of Medicine, Kyoto University and Graduate School of Information Science, Nara Institute of Science and Technology, Nara, Japan, he is currently the associate professor in Biomedical Engineering Lab. at Graduate School of Informatics, Kyoto University, Kyoto, Japan. His research interests include biomedical engineering, computer assisted surgery, virtual reality and medical image computing.

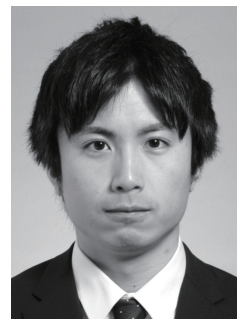

Shimpei Aso received the M.S. degree in informatics from Kyoto University, Kyoto, Japan, in 2016. He is currently at Fourth Financial Sector, NTT DATA Corporation, Japan, where he is involved in research and development for artificial intelligence systems in the evolutional IT center.

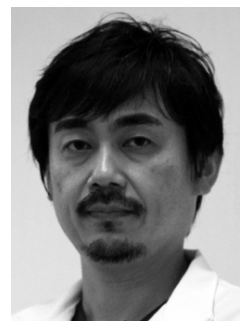

Yuichiro Imai received the Ph.D. degree in medical science at the Nara Medical University, Japan, in 2001. Since Feb 2002, he had been an Assitant Professor in Oral and Maxillofacial Surgery (OMFS) at Nara Medical University. Currently, he works in the department of Oral and Maxillofacial Surgery, Rakuwakai Otowa Hospital, Kyoto, Japan. His main research interests are in fibular transfer surgery, mandibular reconstruction, and medical application of this research. 


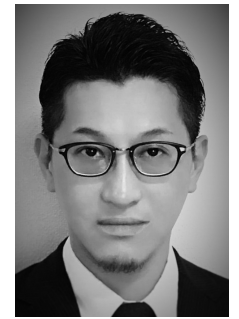

Nobuhiro Ueda graduated from the Department of dentistry of Asahi University, Gifu, Japan. He has continued his research at Department of Oral and Maxillofacial Surgery, Nara Medical University, Nara, Japan, from 2005. His clinical research focuses on head and neck cancer. Currently, he has been in Research association in Oral and Maxillofacial Surgery at Nara Medical University. He is interested in Computer Assisted Surgery, 3-dimensional finite element analysis for oral and maxillofacial reconstructive surgery.

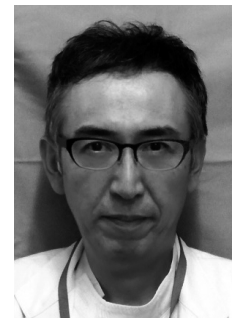

Toshihide Hatanaka graduated from the school and the graduate school of dental technology of Osaka Dental University, Osaka, Japan. He spent as the dental technician at Kagawa Medical University Hospital, Kagawa, Japan. After that, he has worked at Nara Medical University Hospital, Nara, Japan, from 1984. He has become the Vice-chief engineer in 2015 . He is interested in surgical simulation of oral and maxillofacial reconstructive surgery.

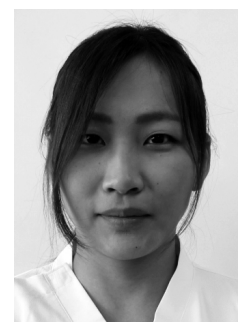

Mao Shiba graduated from the Department of dentistry of Hiroshima University, Hiroshima, Japan. She has worked as the dental technician at Nara Medical University Hospital, Nara, Japan, from 2012. She is interested in the face-guard after treatments of maxillofacial injuries.

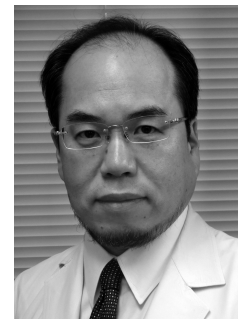

Tadaaki Kirita graduated from Osaka Dental University, Osaka, Japan in 1983 and the graduate school of Nara Medical University, Nara, Japan in 1987. He spent his fellowship at Nara Medical University Hospital and received the training of oral cancer treatments in Saitama Cancer Center Japan from 1991 to 1992 and Memorial SloarnKettering Cancer Center, New York from 1998 to 1999. Prof. Kirita is now the Chief of Dept. of Oral and Maxillofacial Surgery, Nara Medical University from 2002. He is an executive director of Japanese Society of Oral Oncology and directors of Japanese Society of Oral and Maxillofacial Surgeons and Japan Society for Head and Neck Cancer. Prof Kiritafs major interest is in oral oncology and reconstructive surgery of oral and maxillofacial region.

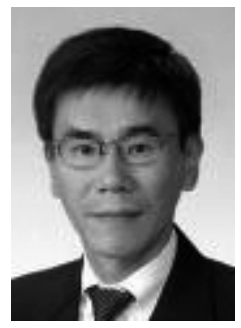

Tetsuya Matsuda received the M.D. degree from Faculty of Medicine, Kyoto University, Kyoto in 1981 and received the $\mathrm{Ph} . \mathrm{D}$. degree from Graduate School of Medicine, Kyoto University in 1988. After working at Third Division, Department of Internal Medicine, Kyoto University Hospital, Division of Cardiovascular Disease, University of Alabama at Birmingham, and Department of Medical Informatics, Kyoto University Hospital, he serves as Professor, Department of Systems Sciences, Graduate School of Informatics, Kyoto University from 2000.

His research field covers magnetic resonance imaging, medical image processing and cardiac simulation. 